\title{
Neurodegenerative diseases: phenome to genome analysis
}

\begin{abstract}
Genome-Wide Association Studies (GWAS) tools are an attractive starting point for disease target discovery. Genetic association evidence provides a strong link to disease phenotypes across diverse human DNAs. The National Center for Biotechnology Information (NCBI) Phenome-Genome Integrator tool (PheGenI) was used to identify genes associated with neurodegenerative disease phenotypes (NeuroGenes). Four hundred ninty-nine known proteins and 30 uncharacterized proteins were found to be associated with diverse neurological diseases including Alzeheimer's, amyotrophic lateral sclerosis (ALS), epilepsy, multiple scelerosis, stroke and Parkinson's. The NeuroGenes also were associated with other diseases and associated disorders. Using diverse bioinformatics and proteomics tools, an atlas of the NeuroGenes was created to facilitate rational biomarker and drug target discovery. NeuroGenes protein expression was detected in diverse body fluids. The NeuroGenes were categorized into functional classes of proteins using protein motif and domains analysis tools. Expression Quantitative Trait Loci (eQTL) analysis identified single nucleotide polymorphisms (SNPs) associated with mRNA expression across diverse sets of samples from Alzheimer's disease, Parkinson's disease, brain infarction, multiple sclerosis, schizophrenia, stroke, mental retardation and neurological cancers. Thirty uncharacterized proteins encompassing structural protein, translation initiation factor, GDP/GTP exchange factor (GEF), transmembrane proteins, tubulin/histonebinding and a lysosomal hydrolase were identified as putative proteins for dementia, Parkinson's, neurobehavior, multiple scelerosis and ALS. These results may provide new biomarkers for neurological diseases.
\end{abstract}

Keywords: Neurological Diseases; Bioinformatics; Proteomics; Genetic Association; Body Fluids; Phenome Analysis; Human Genome; Human Proteome Map; Druggable Targets; Biomarkers; NeuroGenes
Volume 2 Issue I - 2015

\author{
Ramaswamy Narayanan \\ Department of Biological Sciences, Charles E. Schmidt College \\ of Science, Florida Atlantic University, USA
}

\begin{abstract}
Correspondence: Ramaswamy Narayanan, Department of Biological Sciences, Charles E. Schmidt College of Science, Florida Atlantic University, 777 Glades Road, Boca Raton, FL 33431, USA, Tel + |56|2972247, Fax + |56|2973859, Email rnarayan@fau.edu
\end{abstract}

Received: November 24, 2014 | Published: December 18, 2014
Abbreviations: ClinVar, clinical variations; FDA, federal drug administration; eQTL, expression quantitative trait loci; GAD, genetic association database; GtEx, genotype-tissue expression; HapMap, haploid map; HGNC, human genome nomenclature committee; MOPED, model organism protein expression database; PheGenI, phenotype-genotype integrator; ORF, open reading frame; RefSeq, reference sequence; SNP, single nucleotide polymorphism

\section{Introduction}

Neurodegenerative diseases comprise complex sets of disorders which strike primarily in mid- to late-life; their incidence is expected to soar as the population ages. According to the Alzheimer's Association's statistics, five million Americans suffer from Alzheimer's disease. The risk of developing Alzheimer's disease increases with age, and about one in 8 people over the age of 65 is affected by Alzheimer's disease. It is the most common form of mental decline or dementia in older adults.

Further, 1 million people suffer from Parkinson's disease, 400,000 from multiple sclerosis (MS), 30,000 from amyotrophic lateral sclerosis (ALS or Lou Gehrig's disease), and 30,000 from Huntington's disease. ${ }^{1}$ Novel molecular targets are needed to develop a better understanding of these diseases for diagnosis and treatment.

Genome-Wide Association Studies (GWAS) offer clues to assessing risk of development, susceptibility and cures to diverse neurodegenerative diseases. ${ }^{1-10}$ Identifying molecular targets from the GWAS databases offers an improved starting point since a great deal of patient-derived association evidence is readily available. Further, information related to the Copy Number Variations (CNV), gene expression at mRNA levels and the Expression Quantitative Trait Loci (eQTL) across the 1000 Genomes Project from these databases establishesa stronger correlation of a gene target in the pathology of the disease.

The National Center for Biotechnology Information (NCBI) Phenome-Genome Integrator (PheGenI) tool merges National Human Genome Research Institute (NHGRI) GWAS data with several databases, including Gene, database of Genotypes and Phenotypes (dbGaP), Online Mendelian Inheritance in Man (OMIM), the Genotype-Tissue Expression project (GTEx) and the Single Nucleotide Polymorphism database (dbSNP). This phenotype-oriented resource facilitates follow-up studies from GWAS and allows prioritization of variants. ${ }^{11}$ Increasingly, bioinformatics and proteomics approaches are becoming important to target discovery for complex diseases such as neurodegenerative diseases. ${ }^{12-16}$

The availability of protein expression datasets from the Multi Omics Protein Expression Database (MOPED), ${ }^{17}$ the Human Proteome $\mathrm{Map}^{18}$ and the Proteomics $\mathrm{DB}^{19}$ for the human proteome has greatly facilitated the target validation process for biomarker discovery. These datasets encompass protein expression data from diverse tissues as well as body fluids. 
Using a streamlined bioinformatics and proteomics approach, we have recently demonstrated mining of the human genome for target discovery in diseases including cancer and diabetes. ${ }^{20-22}$ In this study, the PheGenI tool was used to develop an initial list of genetically associated single nucleotide polymorphic (SNP) loci with neurological traits. The associated genes (NeuroGenes) were subjected to comprehensive bioinformatics and proteomics analyses $(n=499)$. The NeuroGenes were organized into functional classes of proteins such as enzymes, transporters and extracellular proteins and expression in diverse body fluids was established using protein expression databases. Functional annotation of thirty uncharacterized proteins predicts putative targets for further studies to aid in neurodegenerative disease research.

\section{Materials and methods}

\section{Genome analysis}

The genome analysis was performed using the Genetic Association Disease (GAD) database, ${ }^{23,24}$ the UCSC Genome Browser from the University of California Santa cruz, ${ }^{25}$ the Ensembl Genome Browser, ${ }^{26}$ the National Center for Biotechnology Information, NCBI Gene Expression Quantitative Loci Browser, eQTL ${ }^{11}$ and the Genotype-Tissue Expression Project, GTEx. ${ }^{27}$ The GAD database was downloaded and stored locally. Excel advanced filtering option was used to functionally annotate neurologically associated genes and a separate working database was generated for further manipulation.

\section{Transcriptome analysis}

The transcriptome analysis of the uncharacterized proteins was undertaken using the NCBI-UniGene, SAGE Digital Anatomical Viewer ${ }^{28}$ and the Array Express. ${ }^{29}$

\section{Proteome analysis}

The proteome of the uncharacterized ORFs was analyzed using the UniProt Knowledge Base, UniProtKB, ${ }^{30}$ the Swiss Institute of Bioinformatics (SIB) ExPASy Server's Protein Database (PDB) and post-translational modification sites at ExPASy. ${ }^{31}$ Protein motifs and domains were analyzed using the NCBI Conserved Domain Database, CDD ${ }^{32}$ the PFAM, ${ }^{33}$ ProDom, ${ }^{34}$ InterProscan $4,{ }^{35}$ HMMER $^{36}$ and Signal P Server. ${ }^{37}$ Protein expression batch analysis was performed using the Human Protein Atlas, HPA, ${ }^{38}$ the Multi Omics Proteins Expression Database, MOPED, ${ }^{39}$ the Human Protopedia Reference Database, HPRD ${ }^{40}$ the Human Proteome Map ${ }^{18,41}$ and Proteomes database, proteomics $\mathrm{dB} .{ }^{19}$ For the batch analysis, a comma-separated values (CSV) file of the NeuroGenes list was first generated for use with the protein expression analyses tools indicated above.

\section{Knowledge-based data mining}

Protein and genome knowledge databases used included GeneCards, ${ }^{42}$ the MalaCards ${ }^{43}$ Online Mendelian Inheritance in Man (OMIM), Human Genome Nomenclature Committee, HGNC, ${ }^{44}$ Gene Ontology, Amigo, ${ }^{45}$ NCBI SNP database, the NCBI PhenomeGenome integrator, PheGenI, ${ }^{11}$ the Expression Quantitative Trait Browser, eQTL, ${ }^{27}$ the NCBI Clinical Variations database, ClinVar, ${ }^{46}$ the KEGG pathway and the DAVID functional annotation tool ${ }^{47}$ For PheGenI tool the P-value was set at values $<10-5$ and the R-Squared at 0.3 . For Clinical Variations, the variation filter was set at pathogenic or risk factor associated single nucleotide polymorphisms.

\section{GeneALaCart batch analysis}

The GeneALaCart Meta analysis tool from the GeneCards
(LifeMap Discovery) was used to batch analyse the NeuroGenes for gene names, aliases and descriptions, gene ontology, protein motif and domains, pathways and interactome analysis and drug bank hits.

\section{Data analysis}

The entire database of GAD, Human Protein Atlas, HPA and UniGene was downloaded and the Excel filtering tool was used to scan for the ORFs. Batch analysis of the ORF database was performed for canSar, the MOPED, the DAVID annotation tool, the Human Proteome Map, Proteomics dB, the PheGenI and the eQTL browser.

All of the bioinformatics mining was verified by two independent experiments. For the bioinformatics tools used in the study, the basic parameters indicated by the tool's browser were used. Only statistically significant results per each tool's requirement are reported. Big data from the experiments was first generated as tab-separated values file and was imported into a working Excel document. The Excel data was filtered using the advanced filtering options to create annotated clusters. Prior to using a bioinformatics tool, a series of control query sequences was tested to evaluate the predicted outcome of the results. ${ }^{20-22,48}$

\section{Results}

\section{Neurodegenerative disease-associated traits}

Neurologically relevant gene-related information exists in different databases, which complicates efforts to identify targets for further studies. ${ }^{49-51}$ In order to facilitate mining the human genome for neuro targets, the NCBI PheGenI was analyzed for traits associated with nervous system diseases.

Polymorphic traits, SNPs $(n=1,034)$ associated with diverse neurological diseases and disorders were identified (Figure 1). These SNPs were present in 499 genes encompassing both the known and novel, uncharacterized protein open reading frames (ORFs). Alzheimer's Disease, mental competency, multiple sclerosis and Parkinson's disease harbored the largest number of polymorphic SNPs and associated genes (Supplemental Table S1).

Reasoning that a comprehensive knowledgebase of the 499 genes associated with neurological disease (NeuroGenes) would facilitate target discovery, a comprehensive bioinformatics and proteomics analysis was undertaken. The GeneCards and DAVID Functional Annotation Meta Analysis tools were used to develop the knowledgebase, which encompasses protein identifiers, gene ontology, summary of function, protein motif and domain related information and pathways (Supplemental Table S2).

\section{Neurogenes associated with diverse diseases}

Gene function is often involved across multiple cell types eliciting cell type-specific networks of gene interactions. Identification of gene targets related to multiple diseases offers an advantage of benefitting the drug discovery rationale for therapy for numerous diseases and related disorders. Hence, the involvement of NeuroGenes both in neurologically related and unrelated diseases was next investigated from the NCBI PheGenI data (Figure 2). The NeuroGenes SNPs were also found to be associated with diverse diseases including allergy, cancer, cardiovascular, inflammation, macular degeneration and infections (Supplemental Table S3). Cancer (including astracytoma, glioblastoma and neuroblastoma) had the largest number of geneassociated evidence $(n=189)$. These results underscore the complexity of genes' involvement in multiple diseases. 


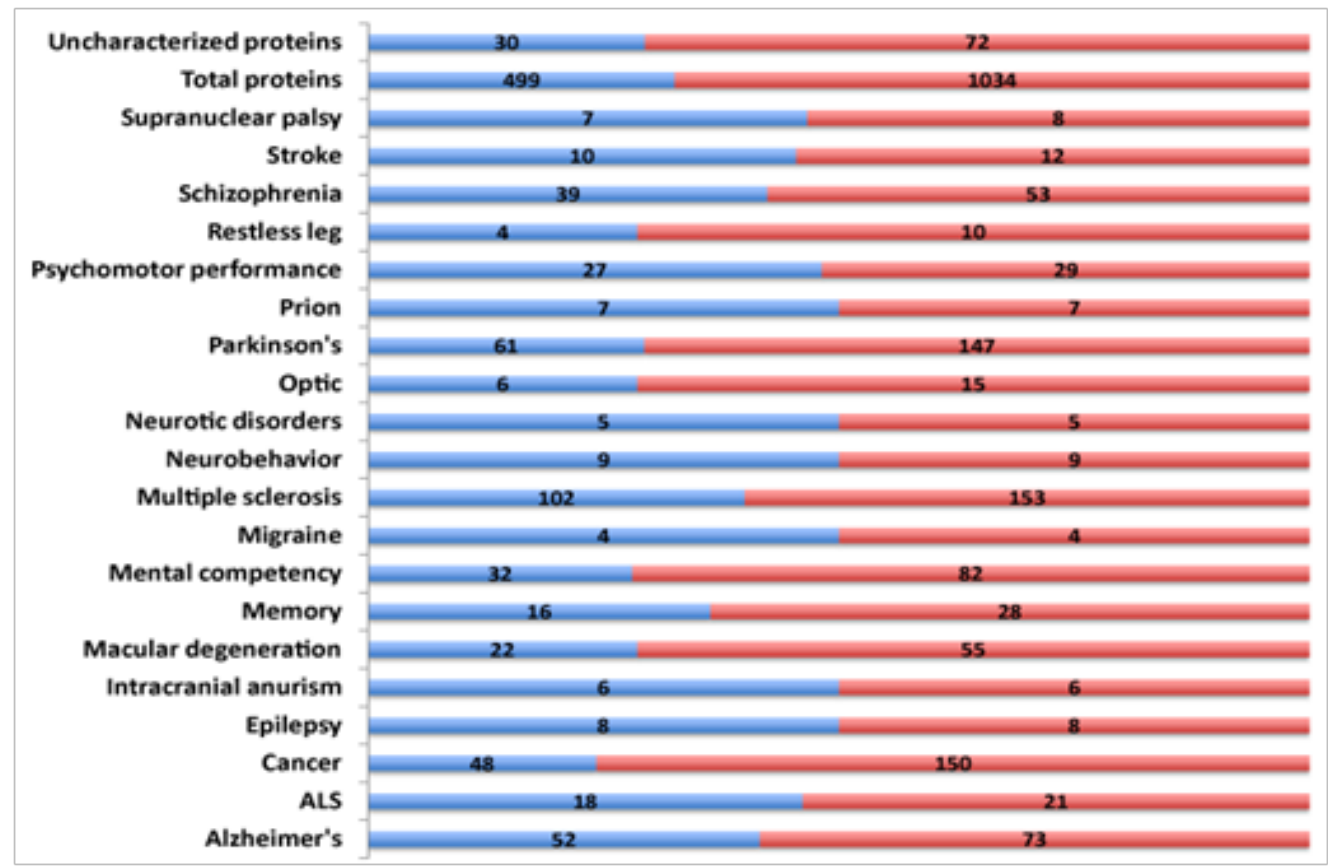

Figure I Phenome-genome association of NeuroGenes. The NCBI Phenome Genome Integrator tool was used to identify neurologically associated polymorphisms. The number of genes (blue) and single nucleotide polymorphisms (red) are shown for indicated neurological traits.

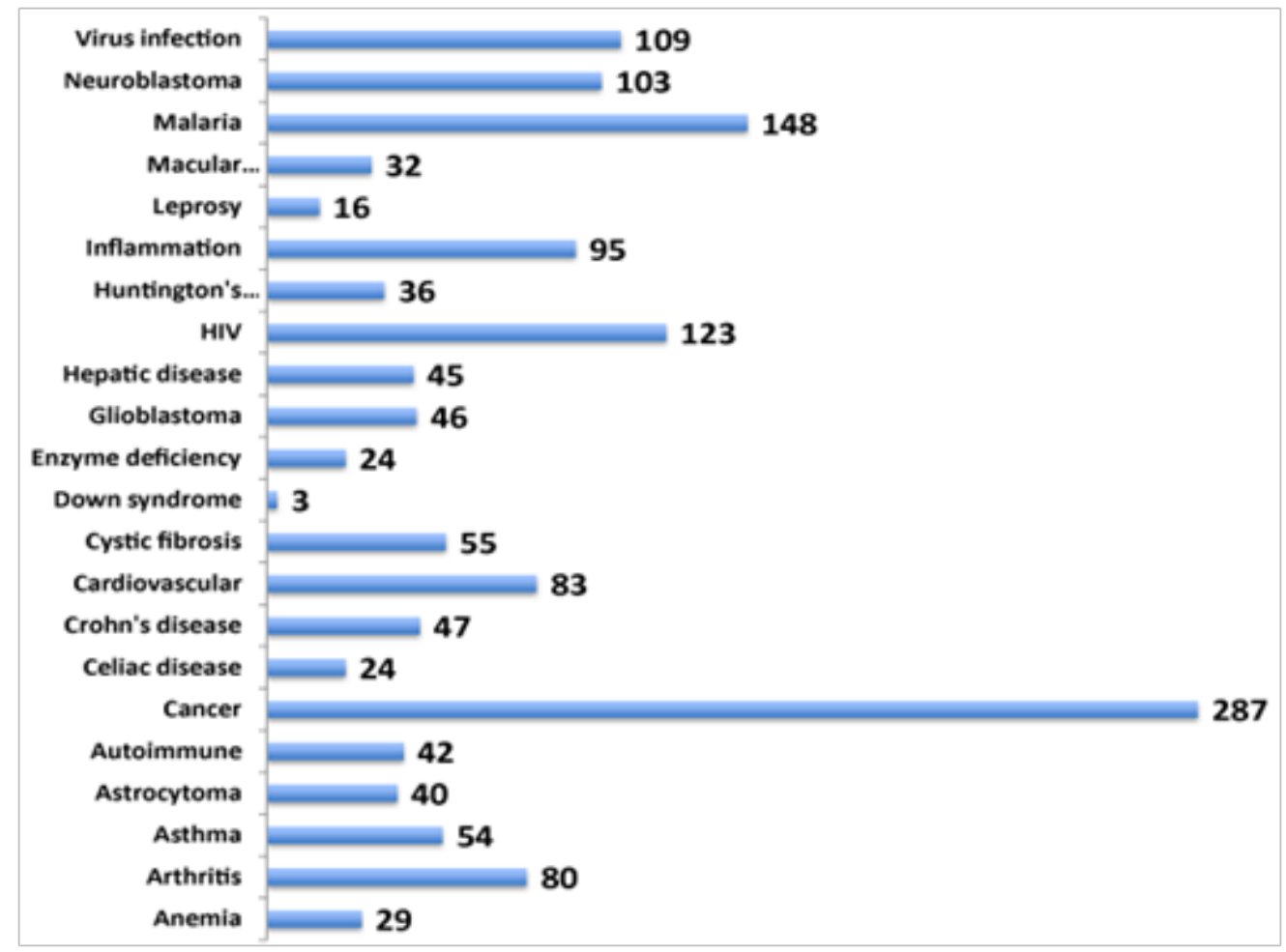

Figure 2 Association of NeuroGenes with other diseases and disorders. The NeuroGenes output from the NCBI Phenome Genome Integrator tool was clustered into association evidence with the indicated diseases and disorders. The numbers indicate the number of genes showing association.

Protein expression of neurogenes in body fluids: and the ProteomicsDB databases. The NeuroGenes protein expression baseline analysis

The recent availability of the human proteome map dataset for protein expression in multiple tissues and body fluids ${ }^{18,19,39}$ enabled this study to develop a comprehensive baseline protein expression dataset on the NeuroGenes (Figure 3). A composite protein expression dataset was generated using the MOPED, the Human Proteome Map was detected in diverse body fluids including ascites, cerebrospinal fluid, milk, saliva, serum, semen and urine. Most of the Neurogenes were detected in the brain, spinal cord tissues and peripheral blood lymphocytes (Supplemental Table S4). This baseline analysis for protein expression in normal human body fluids establishes a framework for biomarker screening efforts for diverse neurological diseases. 


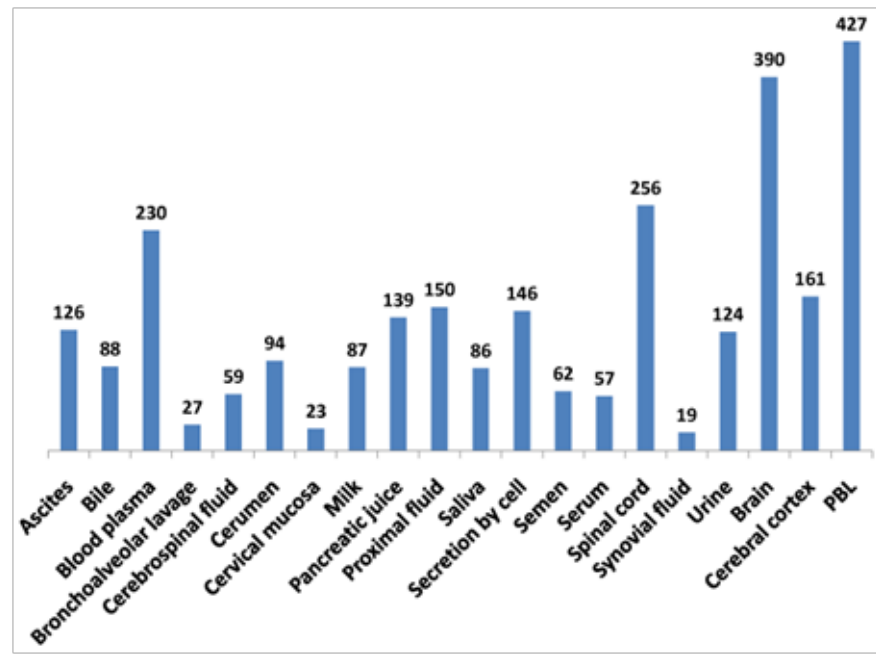

Figure 3 NeuroGene expression profile in body fluids. The protein expression of the NeuroGenes was inferred from the Multi Omics Profiling Expression Database, the Human Proteome Map and the Proteomics databases. The numbers indicate the number of proteins present in the indicated body fluid.

\section{Gene ontology of neurogenes: cell location, function and process}

To add to the NeuroGenes knowledgebase, the Gene Ontology was investigated next using the GeneALaCart Meta Analysis tool (Figure 4). The proteins were classified into cellular location (Panel A), function (Panel B) and processes involved (Panel C). The largest number of proteins was found to be membrane bound (280/499). In addition, extracellular, secreted, nuclear/mitochondrial/Golgi as well as cytoskeletal proteins were inferred (Panel A; also Supplemental Table S5). The NeuroGenes encompassed druggable targets including channel proteins, transporters, receptors and enzymes (Panel B). Enzymes constituted the largest druggable class of proteins (213/499). The NeuroGenes were implicated in diverse biological processes such as angiogenesis, apoptosis, axon, differentiation and development, immune function, neuronal migration and signal transduction (Panel C). The largest number of NeuroGenes was associated with regulation of gene expression (247/499).



Figure 4 Gene ontology analysis of the NeuroGenes. The cellular location (Panel A), the function (Panel B) and the process (Panel C) of the NeuroGenes was inferred from the GeneALaCart and the DAVID functional annotation tool. The number of genes for indicated category is shown.

\section{Neurogene pathways}

Elucidating the pathways implicated in the expression of the
NeuroGenes is essential to deriving functional knowledge for further studies. The pathway-related information was inferred from the KEGG and BioCarta pathway analyses tools (Figure 5). Diverse pathways such as addiction, AKT/MAPK signaling, Alzheimer's, inflammation, infections, metabolic, prion, STAT signaling and vesicular trafficking were involved with the NeuroGenes (Supplemental Table S6).

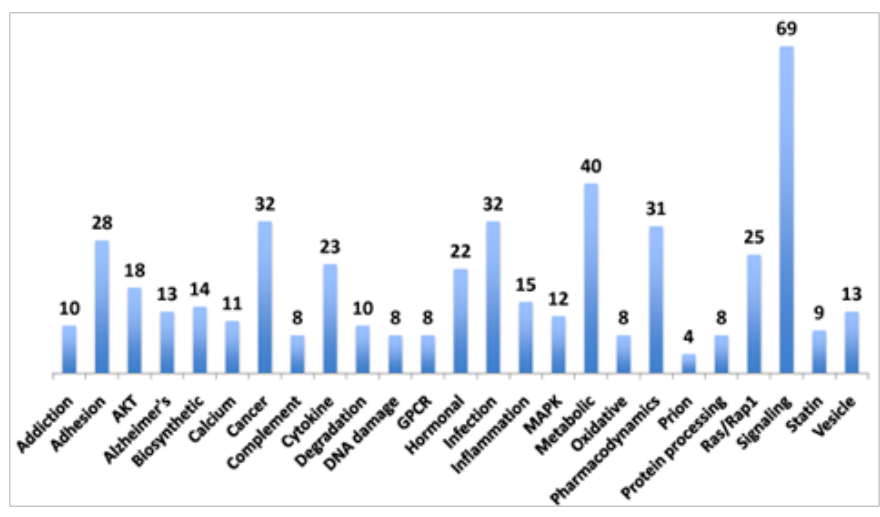

Figure 5 Pathway mapping of the NeuroGenes. The NeuroGenes pathways were identified using the Human Genome Nomenclature Committee pathways, the KEGG pathway analysis tool and the Biocarta pathway tool. The number of genes involved in the indicated pathways is shown.

\section{Neurogene protein class and families}

Hints regarding function were next investigated using protein motif and domain analyses tools, the HGNC motifs and domains, UniProt Domains, the InterPro, the Signal P server, the Protein Family, PFAM and the DAVID functional annotation tool (Figure 6). The 289 NeuroGenes were classified into adhesion molecules, ligand and metal binding proteins, enzymes, receptors, transporters, channel proteins, immunoglobulins and transcription factors (Supplemental Table S7). These results further verified the possible druggable nature of many NeuroGenes.

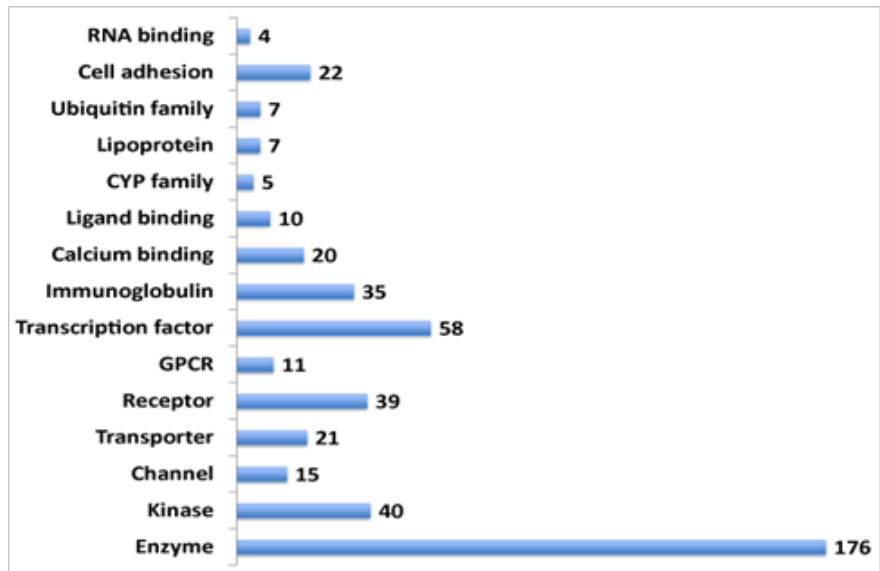

Figure 6 Functional annotation of the NeuroGenes protein classes. The protein classes were predicted using the InterPro domains and motifs, the UniProt Domains, the Signal P server, the Protein Family, the ScanProsite from the Expasy server and the NCBI Conserved Domain database. The number of proteins for the indicated functional class is shown.

\section{Expression quantitative trait loci analysis of the neurogenes}

An expression quantitative trait locus (eQTL) represents a marker (locus) in the genome in which variation between individuals is associated with a quantitative gene expression trait, often measured 
as mRNA abundance. The three components critical to validating eQTL results include i) a SNP marker; ii) the gene expression levels, as measured by a probe or sequence information; and iii) a measure of the statistical association between the two in a study population, such as the P-value. The eQTL browser provides an approach to query the eQTL database. ${ }^{52}$

The eQTLs can be cis, where the genotyped marker is near the expressed gene, or trans, in which the genotyped marker is distant from the expressed gene either in the same or on another chromosome. Currently only the cis-eQTLs ${ }^{53}$ are available. In order to establish neurological disease-associated eQTL results for the NeuroGenes, the PheGenI tool was used to batch analyze the SNPs associated with the NeuroGenes. Genotypes for the NeuroGenes were selected for exons, introns, near gene and Untranslated Region (UTR)

From these population studies, 18 NeuroGenes were found to have eQTL association with diverse neurological diseases (Table 1 Included as supplementary). The SNPs for these 18 genes were present in diverse human DNAs from the 1000 Genomes Project. The diseases showing associated eQTL genes included Alzheimer's disease (HLA-A, MAPK8IP1, MAPT), dementia (ITIH4), multiple scelerosis (CLECL1, FAM119B, HLA-DQB1, HLA-G and RPS6KB1), natriuretic peptide (NFIA), neuroblastoma (BARD1, RPS6KB2), neural tube defect (MTHFR), Parkinson's disease (LRRC37A, MAPK8IP1 and MAPT), schizophrenia (AHI1, HLADQA1 and HLA-A), stroke (HLA-DQA1), prion disease (MAPT and HLA-DQB1) and retinoblastoma (HLA-DRA).

The ribosomal protein S6 kinase, 70kDa, polypeptide 1 (RPS6KB1) was associated with diverse neuronal cancers including malignant peripheral nerve sheath tumor, neuroendocrine tumor, glioblastoma multiforme, malignant glioma, astrocytoma, neuroblastoma and neuronitis. The Genotype-Tissue Expression project (GTEx) portal analysis showed that 13/14 eQTL genes were expressed in all tissues analyzed (Supplemental Table S8).

The NCBI ClinVar dataset showed clinically relevant SNPs for frontotemporal dementia, systemic lupus, diabetes Type 1 and Type 2 , celiac disease, developmental delay, Grave's disease, hereditary neuroblastoma, risk factors for psoriasis susceptibility 1 , and susceptibility to HIV-1 viremia and Schizophrenia 9 (Table 1 Included as supplementary).

\section{Uncharacterized neurogenes: novel targets}

Recently we have mined the human proteome for uncharacterized proteins to enable novel disease target discovery. Targets relevant to cancer and diabetes were identified. ${ }^{21,22,48,54}$ Reasoning that the 30 uncharacterized proteins, that showed strong genetic association with neurological diseases may offer novel biomarker and target potential, a comprehensive analysis was undertaken (Table 2 Included as supplementary). A structural protein, C1orf125 containing axonal dynin light chain was found to be associated with multiple sclerosis, Huntington's disease and diabetes Type $2 .{ }^{55,56}$

Six Parkinson's disease-associated novel proteins were also identified. These included FAM47E (lncRNA transcription co factor), KIAA0427 (translation initiation factor), KIAA1267 (histone binding), TMEM175 (lysosomal transmembrane protein), C9orf72 (GDP/GTP exchange factor) and a novel enzyme, TMEM55A (transmembrane hydrolase).

The C20orf196 (uncharacterized), FAM110C (lncRNA alphatubulin binding protein) and C9orf5 (transmembrane glycoprotein) were associated with neurobehavioral manifestations. ${ }^{57,58}$ Two of the ORFs were associated with carotid artery diseases, C9orf93, a myosinrelated nucleotide binding protein ${ }^{55}$ and C20orf196 (uncharacterized protein). ${ }^{58}$

A GDP/GTP exchange factor (GEF) showed restricted expression in the adult brain and peripheral blood lymphocytes and was associated with amyotrophic lateral sclerosis, Huntington's, frontotemporal dementia and Parkinson's. ${ }^{59-62}$ A repeat expansion in the C9orf72/ GEF gene has recently been identified as a major cause of familial and sporadic frontotemporal lobar degeneration, Huntington's, Parkinson's and amyotrophic lateral sclerosis. A membrane testisspecific basic protein, C6orf10, showed association with multiple sclerosis ${ }^{63}$ asthma ${ }^{64}$ and rheumatoid arthritis ${ }^{65}$ (Supplementary Table S9). Additional bioinformatics details on these uncharacterized NeuroORFs are shown in the (Supplementary Table S10). These novel proteins offer opportunities for further development of novel diagnostics and therapeutics.

\section{Therapeutic value of the neurogenes}

Drug hits for genes (including chemical compounds and Federal Drug Administration (FDA) approved drugs) are available in diverse drug-related databases. Reasoning that mining for drugs implicated with the pathways associated with the NeuroGenes might offer a framework for understanding mechanism, the NeuroGenes were batch analyzed using the GeneALaCart Meta Analysis tool for drug hits in the drug banks.

Numerous FDA-approved drug hits and well as other compounds which target the NeuroGene's pathways were identified including statins, CyclosporineA,Donepezil, Rosiglitazone(Alzheimer's disease, cognition), antivirals such as ritonavir and Ganciclovir, hormones including Estrogen and Testosterone, Verapamil, Isradipine (gating inhibitor), Diltiazem (Channel blocker), Gemtuzumabozogamicin, Cisplatin and Methotrexate (antineoplastics) and antibiotics such as rapamycin, Tunicamycin (Supplementary Table S11) for complete details).

\section{Discussion}

Diagnosis and therapy of complex neurodegenerative diseases requires novel molecular targets. Current drug targets for Alzheimer's disease, Parkinson's, Huntington's disease and ALS revolve around functional classes of proteins such as protein aggregation, mitochondrion proteins, N-methyl-d-aspartic acid (NMDA) receptors, voltage gated calcium channels (VGCCs), neuronal nitric oxide synthase (nNOS), oxidative stress from reactive oxygen species, and DNA damage repair enzymes. ${ }^{66-68}$

Individuals with neurodegenerative diseases such as Parkinson's or Alzheimer's are currently benefiting from drugs that target a single gene. However, the single agent treatment modality is inefficient. Increasingly it is becoming a paradigm in drug discovery to focus on drug candidates that affect multiple neural and biochemical targets for the treatment of diverse associated disorders such as cognition impairment, motor dysfunction, depression and neurodegeneration. .6,69-71 $^{66}$

Identification of molecular targets from unbiased GWAS databases is likely to contribute to better therapeutic target discovery for neurodegenerative diseases.,72 Further, such targets are likely to be more relevant to diverse populations because of strong genetic association evidence across the global population DNAs. Utilizing a similar rationale, pancreatic cancer targets were recently identified. ${ }^{20}$ 
In this study, neurologically associated traits were enriched based on GWAS evidence and a functional annotation of the classes of proteins was established. The 499 NeuroGenes thus identified showed overlapping association evidence with other diseases and disorders including allergy, cancer, cardiovascular, infections, inflammation and autoimmune disorders. Interestingly, a significant number of these proteins were detected in diverse body fluids such as blood, cerebrospinal fluid, milk, urine, saliva or semen. A strong correlation of protein expression data was seen when using different protein expression databases such as the MOPED and Proteomics DB. This is encouraging as far as the specificity of prediction is concerned, since the tissue samples for these studies came from different patients. These results also provide baseline expression data for these proteins to further the development of novel biomarkers for diverse neurological diseases.

Using gene ontology and protein motif and domain analysis, the NeuroGenes were further categorized into functional classes of proteins. The database of NeuroGenes was sorted into protein classes encompassing druggable genes including enzymes, $G$ protein coupled receptors, transporters, transcription factors and channel proteins. ${ }^{73}$ This should aid in the prioritization of efforts to validate target genes.

The eQTL analysis of the NeuroGenes led to the identification of 18 genes showing strong association with ALS, Alzheimer's, cancer, multiple sclerosis, Parkinson's and schizophrenia. Of interest was the discovery among those identified of RPS6KB1, a member of the ribosomal S6 kinase family of serine/threonine kinases. The encoded protein responds to mTOR (mammalian target of rapamycin) signaling to promote protein synthesis, cell growth, and cell proliferation. This gene is a risk factor for a great number of tumor types including malignant peripheral nerve sheath tumor, neuroendocrine tumor, glioblastoma multiforme, malignant glioma, astrocytoma and neuroblastoma, lymphoma, breast tumors, non-small cell lung carcinoma, in addition to Alzheimer's disease and multiple sclerosis. ${ }^{74-79}$ Consistent with these results, the Catalogue of Somatic Mutation in Cancer database, $\operatorname{COSMIC}^{80}$ was found to harbor a large number of somatic mutations in the protein kinase domain (substitution missense). Moreover, mouse insertional mutagenesis experiments support RPS6KB1 as a driver cancer-causing gene. ${ }^{81,82}$ This kinase offers a novel biomarker and drug therapy opportunity for diverse cancers.

This study also identified 30 uncharacterized proteins with association evidence in multiple sclerosis, Parkinson's disease, Huntington's disease, amyotrophic lateral sclerosis and frontotemporal dementia. The protein classes encompassed included transmembrane glycoprotein, GDP/GTP exchange factor, transcription factor, myosin/ tubulin binding proteins, LDL and nuclear receptors, transporters and a lysosomal transmembrane enzyme. Six of the 30 proteins were associated with Parkinson's disease. It is tempting to suggest that these may offer a fingerprint for Parkinson's disease. Additional verification is warranted.

Analysis of the drug banks identified FDA-approved drugs and as well as compounds implicating diverse biochemical pathways involved with the NeuroGenes. These drugs and compounds provides a starting point for use in model systems and for improved Structure Activity Relationship (SAR) studies in the future.

Identification of proteins as genetically linked using the GWAS dataset provides a strong rationale for lead prioritization in biomarker and therapeutic target research. Such an approach is likely to lead to the development of more relevant targets for further studies.

\section{Conclusion}

The results presented in this study demonstrate that mining the phenome-genome association databases can be an effective approach for disease biomarker and putative target discovery for further study. The 30 novel proteins characterized in this study shed more light on the Dark Matter proteome and offer new opportunities for understanding diverse neurological diseases. The atlas of the NeuroGenes generated in this study can provide a starting point for target verification for biomarker potential and eventual drug therapy use.

\section{Acknowledgements}

This work was supported in part by the Genomics of Cancer Fund, Florida Atlantic University Foundation. I thank Dr. Stein of the GeneCards team for generous permission to use the powerful GeneALaCart tool; Dr. Montague, Kolker Laboratory of the MOPED Team for batch analysis of the ORFs and the Human Proteome Map and ProteomesDB for the datasets. I thank Jeanine Narayanan for editorial assistance.

\section{Conflict of interest}

The author declares no conflict of interest.

\section{References}

1. Checkoway H, Lundin JI, Kelada SN. Neurodegenerative diseases. IARC Sci Publ. 2011;(163):407-419.

2. Gandhi S, Wood NW. Genome-wide association studies: the key to unlocking neurodegeneration? Nat Neurosci. 2010;13(7):789-794.

3. McCarthy MI, Abecasis GR, Cardon LR, et al. Genome-wide association studies for complex traits: consensus, uncertainty and challenges. Nat Rev Genet. 2008;9(5):356-369.

4. Simon-Sanchez J, Singleton A. Genome-wide association studies in neurological disorders. The Lancet Neurol. 2008;7(11):1067-1072.

5. Frazer KA, Murray SS, Schork NJ, et al. Human genetic variation and its contribution to complex traits. Nat Rev Genet. 2009;10(4):241-251.

6. Simon-Sanchez J, Schulte C, Bras JM, et al. Genome-wide association study reveals genetic risk underlying Parkinson's disease. Nat Genet. 2009;41(12):1308-1312.

7. Satake W, Nakabayashi Y, Mizuta I, et al. Genome-wide association study identifies common variants at four loci as genetic risk factors for Parkinson's disease. Nat Genet. 2009;41(12):1303-1307.

8. Pittman AM, Fung HC, de Silva R. Untangling the tau gene association with neurodegenerative disorders. Hum Mol Genet. 2006;15 Spec No 2:R188-R95

9. Tsuji S. Genetics of neurodegenerative diseases: insights from highthroughput resequencing. Hum Mol Genet. 2010;19(R1):R65-R70.

10. Zou F, Chai HS, Younkin CS, et al. Brain expression genome-wide association study (eGWAS) identifies human disease-associated variants. PLoS Genet. 2012;8(6):e1002707.

11. Ramos EM, Hoffman D, Junkins HA, et al. Phenotype-Genotype Integrator (PheGenI): synthesizing genome-wide association study (GWAS) data with existing genomic resources. Eur J Hum Genet. 2014;22(1):144-147.

12. Guffanti A, Simchovitz A, Soreq H. Emerging bioinformatics approaches for analysis of NGS-derived coding and non-coding RNAs in neurodegenerative diseases. Front Cell Neurosci. 2014;8:89.

13. Nguyen TP, Caberlotto L, Morine MJ, et al. Network analysis of neurodegenerative disease highlights a role of Toll-like receptor signaling. BioMed Res Int. 2014;2014:686505. 
14. Zhang J, Keene CD, Pan C, et al. Proteomics of human neurodegenerative diseases. J Neuropathol Exp Neurol. 2008;67(10):923-932.

15. Hwang $\mathrm{H}$, Zhang J, Chung KA, et al. Glycoproteomics in neurodegenerative diseases. Mass Spectrom Rev. 2010;29(1):79-125.

16. Kroksveen AC, Opsahl JA, Aye TT, et al. Proteomics of human cerebrospinal fluid: discovery and verification of biomarker candidates in neurodegenerative diseases using quantitative proteomics. J Proteomics. 2011;74(4):371-388

17. Kim SM, Wang JW. Calcium imaging of pheromone responses in the insect antennal lobe. Methods Mol Biol. 2013;1068:179-187.

18. Kim MS, Pinto SM, Getnet D, et al. A draft map of the human proteome. Nature. 2014;509(7502):575-581.

19. Wilhelm M, Schlegl J, Hahne H, et al. Mass-spectrometry-based draft of the human proteome. Nature. 2014;509(7502):582-587.

20. Narayanan R. Phenome-Genome association studies of pancreatic cancer: New targets for therapy and diagnosis. Cancer genomics \& proteomics. 2014.

21. Delgado A, Brandao P, Chapado M, et al. Open Reading Frames Associated with Cancer in the Dark Matter of the Human Genome. Cancer Genomics Proteomics. 2014;11(4):201-213.

22. Delgado A, Brandao, P, Narayanan, R. Diabetes Associated Genes from the Dark Matter of the Human Proteome. MOJ Proteomics Bioinform. 2014;1(4):00020.

23. Becker $\mathrm{KG}$, Barnes $\mathrm{KC}$, Bright $\mathrm{TJ}$, et al. The genetic association database. Nat Genet. 2004;36(5):431-432.

24. Zhang Y, De S, Garner JR, et al. Systematic analysis, comparison, and integration of disease based human genetic association data and mouse genetic phenotypic information. BMC Med Genomics. 2010;3:1

25. Karolchik D, Barber GP, Casper J, et al. The UCSC Genome Browser database:2014 update. Nucleic Acids Res. 2014;42(Database issue):D764-D770.

26. Flicek P, Amode MR, Barrell D, et al. Ensembl 2014. Nucleic Acids Res. 2014;42(Database issue):D749-D755.

27. Consortium GTEx. The Genotype-Tissue Expression (GTEx) project. Nat Genet. 2013;45(6):580-585.

28. Velculescu VE, Zhang L, Vogelstein B, et al. Serial analysis of gene expression. Science. 1995;270(5235):484-487.

29. Parkinson H, Sarkans U, Shojatalab M, et al. ArrayExpress-a public repository for microarray gene expression data at the EBI. Nucleic Acids Res. 2005;33(Database issue):D553-D555.

30. UniProt C. Update on activities at the Universal Protein Resource (UniProt) in 2013. Nucleic Acids Res. 2013;41(Database issue):D43D47.

31. Artimo P, Jonnalagedda M, Arnold K, et al. ExPASy: SIB bioinformatics resource portal. Nucleic Acids Res. 2012;40(Web Server issue):W597W603.

32. Marchler-Bauer A, Zheng C, Chitsaz F, et al. CDD: conserved domains and protein three-dimensional structure. Nucleic Acids Res 2013;41(Database issue):D348-D352.

33. Finn RD, Bateman A, Clements J, et al. Pfam: the protein families database. Nucleic Acids Res. 2014;42(D1):D222-D230.

34. Servant F, Bru C, Carrere S, et al. ProDom: automated clustering of homologous domains. Brief Bioinfom. 2002;3(3):246-251.

35. Hunter S, Apweiler R, Attwood TK, et al. InterPro: the integrative protein signature database. Nucleic Acids Res. 2009;37(Database issue):D211D215.
36. Finn RD, Clements J, Eddy SR. HMMER web server: interactive sequence similarity searching. Nucleic Acids Res. 2011;39(Web Server issue):W29-W37.

37. Petersen TN, Brunak S, von Heijne G, et al. Signal P 4.0: discriminating signal peptides from transmembrane regions. Nat Methods. 2011;8(10):785-786.

38. Uhlen M, Oksvold P, Fagerberg L, et al. Towards a knowledge-based Human Protein Atlas. Nat Biotechnol. 2010;28(12):1248-1250.

39. Kolker E, Higdon R, Haynes W, et al. MOPED: Model Organism Protein Expression Database. Nucleic Acids Res. 2012;40(Database issue):D1093-D1099.

40. Mathivanan S, Ahmed M, Ahn NG, et al. Human Proteinpedia enables sharing of human protein data. Nat Biotechnol. 2008;26(2):164-167.

41. Maruyama Y, Kawamura Y, Nishikawa T, et al. HGPD: Human Gene and Protein Database, 2012 update. Nucleic Acids Res. 2012;40(Database issue):D924-D929.

42. Safran M, Dalah I, Alexander J, et al. GeneCards Version 3:the human gene integrator. Database (Oxford). 2010;2010:baq020.

43. Rappaport N, Nativ N, Stelzer G, et al. MalaCards: an integrated compendium for diseases and their annotation. Database (Oxford) 2013;2013:bat018.

44. Zhang Y. I-TASSER server for protein 3D structure prediction. BMC Bioinformatics. 2008;9:40.

45. Ashburner M, Ball CA, Blake JA, et al. Gene ontology: tool for the unification of biology. The Gene Ontology Consortium. Nat Genet. $2000 ; 25(1): 25-29$

46. Landrum MJ, Lee JM, Riley GR, et al. ClinVar: public archive of relationships among sequence variation and human phenotype. Nucleic Acids Res. 2014;42(Database issue):D980-D985.

47. Huang DW, Sherman BT, Lempicki RA. Systematic and integrative analysis of large gene lists using DAVID bioinformatics resources. Nat Protoc. 2008;4(1):44-57.

48. Delgado AP, Hamid S, Brandao P, et al. A novel transmembrane glycoprotein cancer biomarker present in the $\mathrm{x}$ chromosome. Cancer Genomics Proteomics. 2014;11(2):81-92.

49. Taccioli C, Tegner J, Maselli V, et al. ParkDB: a Parkinson's disease gene expression database. Database (Oxford). 2011;2011:bar007.

50. Na D, Rouf M, O'Kane CJ, et al. NeuroGeM, a knowledgebase of genetic modifiers in neurodegenerative diseases. BMC Med Genomics. 2013;6:52

51. Vasaikar SV, Padhi AK, Jayaram B, et al. NeuroDNet - an open source platform for constructing and analyzing neurodegenerative disease networks. BMC Neurosci. 2013;14:3.

52. Liang L, Morar N, Dixon AL, et al. A cross-platform analysis of 14,177 expression quantitative trait loci derived from lymphoblastoid cell lines. Genome Res. 2013;23(4):716-726.

53. Stranger BE, Montgomery SB, Dimas AS, et al. Patterns of cis regulatory variation in diverse human populations. PLoS Genet. 2012;8(4):e1002639.

54. Delgado A, Brandao P, Hamid S, et al. Mining the Dark Matter of the Cancer Proteome for novel biomarkers. Current Cancer Therapy Reviews. 2013;9(4):265-277.

55. Satoh J, Kawana N, Yamamoto Y. Pathway Analysis of ChIP-Seq-Based NRF1 Target Genes Suggests a Logical Hypothesis of their Involvement in the Pathogenesis of Neurodegenerative Diseases. Gene Regul Syst Bio. 2013;7:139-152 
56. Bailey SD, Xie C, Do R, et al. Variation at the NFATC2 locus increases the risk of thiazolidinedione-induced edema in the Diabetes REduction Assessment with ramipril and rosiglitazone Medication (DREAM) study. Diabetes care. 2010;33(10):2250-2253.

57. Xu C, Aragam N, Li X, et al. BCL9 and C9orf5 are Associated with Negative Symptoms in Schizophrenia: Meta-Analysis of Two GenomeWide Association Studies. PloS ONE. 2013;8(1):e51674.

58. Luciano M, Hansell NK, Lahti J, et al. Whole genome association scan for genetic polymorphisms influencing information processing speed. Biol Psychol. 2011;86(3):193-202.

59. Farg MA, Sundaramoorthy V, Sultana JM, et al. C9ORF72, implicated in amytrophic lateral sclerosis and frontotemporal dementia, regulates endosomal trafficking. Hum Mol Genet. 2014;23(13):3579-3595.

60. Le Ber I, Camuzat A, Guillot-Noel L, et al. C9ORF72 repeat expansions in the frontotemporal dementias spectrum of diseases: a flow-chart for genetic testing. J Alzheimers Dis. 2013;34(2):485-499.

61. Hensman Moss DJ, Poulter M, Beck J, et al. C9orf72 expansions are the most common genetic cause of Huntington disease phenocopies. Neurology. 2014;82(4):292-299.

62. Luigetti M, Quaranta D, Conte A, et al. Frontotemporal dementia, Parkinsonism and lower motor neuron involvement in a patient with C9ORF72 expansion. Amyotroph Lateral Scler Frontotemporal Degener. 2013;14(1):66-69.

63. Martinelli-Boneschi F, Esposito F, Brambilla P, et al. A genomewide association study in progressive multiple sclerosis. Mult Scler. 2012;18(10):1384-1394.

64. Hirota T, Takahashi A, Kubo M, et al. Genome-wide association study identifies three new susceptibility loci for adult asthma in the Japanese population. Nat Genet. 2011;43(9):893-896.

65. Stahl EA, Raychaudhuri S, Remmers EF, et al. Genome-wide association study meta-analysis identifies seven new rheumatoid arthritis risk loci. Nat Genet. 2010;42(6):508-514.

66. Youdim MB, Buccafusco JJ. Multi-functional drugs for various CNS targets in the treatment of neurodegenerative disorders. Trends Pharmacol Sci. 2005;26(1):27-35.

67. Hilbush BS, Morrison JH, Young WG, et al. New prospects and strategies for drug target discovery in neurodegenerative disorders. NeuroRx. 2005;2(4):627-637.

68. Trippier PC, Jansen Labby K, Hawker DD, et al. Target- and mechanismbased therapeutics for neurodegenerative diseases: strength in numbers. $J$ Med Chem. 2013;56(8):3121-3147.
69. Talwar P, Silla Y, Grover S, et al. Genomic convergence and network analysis approach to identify candidate genes in Alzheimer's disease. BMC Genomics. 2014;15:199.

70. Dixon SJ, Stockwell BR. Identifying druggable disease-modifying gene products. Curr Opin Chem Biol. 2009;13(5-6):549-555.

71. do Carmo Costa M, Paulson HL. New hope for therapy in neurodegenerative diseases. Cell Res. 2013;23(10):1159-1160.

72. Ramanan VK, Saykin AJ. Pathways to neurodegeneration: mechanistic insights from GWAS in Alzheimer's disease, Parkinson's disease, and related disorders. Am J Neurodegener Dis. 2013;2(3):145-175.

73. Hopkins AL, Groom CR. The druggable genome. Nat Rev Drug Discov. 2002;1(9):727-730.

74. Zhao XF, Zhao MY, Chai L, et al. Amplified RPS6KB1 and CDC2 genes are potential biomarkers for aggressive $\mathrm{HIV}+\mathrm{EBV}+$ diffuse large B-cell lymphomas. Int J Clin Exp Pathol. 2013;6(2):148-154.

75. Li PD, Zhang WJ, Zhang MY, et al. Overexpression of RPS6KB1 predicts worse prognosis in primary HCC patients. Med Oncol. 2012;29(5):3070 3076.

76. Slattery ML, Lundgreen A, Herrick JS, et al. Genetic variation in RPS6KA1, RPS6KA2, RPS6KB1, RPS6KB2, and PDK1 and risk of colon or rectal cancer. Mutat Res. 2011;706(1-2):13-20.

77. Zhang Y, Ni HJ, Cheng DY. Prognostic value of phosphorylated mTOR/ RPS6KB1 in non- small cell lung cancer. Asian Pac J Cancer Prev. 2013;14(6):3725-3728.

78. International Multiple Sclerosis Genetics C, Wellcome Trust Case Control C, Sawcer S, et al. Genetic risk and a primary role for cell-mediated immune mechanisms in multiple sclerosis. Nature. 2011;476(7359):214219.

79. International Multiple Sclerosis Genetics C, Lill CM, Schjeide BM, et al. MANBA, CXCR5, SOX8, RPS6KB1 and ZBTB46 are genetic risk loci for multiple sclerosis. Brain. 2013;136(Pt 6):1778-1782.

80. Forbes SA, Bindal N, Bamford S, et al. COSMIC: mining complete cancer genomes in the Catalogue of Somatic Mutations in Cancer Nucleic Acids Res. 2011;39(Database issue):D945-D950.

81. Ranzani M, Annunziato S, Adams DJ, et al. Cancer gene discovery: exploiting insertional mutagenesis. Mol Cancer Res. 2013;11(10):11411158.

82. Mann KM, Ward JM, Yew CC, et al. Sleeping Beauty mutagenesis reveals cooperating mutations and pathways in pancreatic adenocarcinoma. Proceedings of the National Academy of Sciences of the United States of America. 2012;109(16):5934-5941. 